\begin{tabular}{|c|c|}
\hline Title & Repeatability of Rest and Hyperemic Myocardial Blood Flow Measurements with 82Rb Dynamic PET \\
\hline Author(s) & Manabe, Osamu; Y oshinaga, Keiichiro; Katoh, Chietsugu; Naya, Masanao; deKemp, Robert A .; Tamaki, Nagara \\
\hline Citation & $\begin{array}{l}\text { Journal of Nuclear Medicine, 50(1), } 68.71 \\
\text { https://doi.org/10.2967//numed.108.055673 }\end{array}$ \\
\hline Issue Date & 2009 \\
\hline Doc URL & http:/hdl.handle.net/2115/46752 \\
\hline Rights & Reprinted by permission of the Society of Nuclear Medicine \\
\hline Type & article \\
\hline File Information & 50_1_68.pdf \\
\hline
\end{tabular}

Instructions for use 


\section{Repeatability of Rest and Hyperemic Myocardial Blood Flow Measurements with ${ }^{82}$ Rb Dynamic PET}

Osamu Manabe ${ }^{1}$, Keiichiro Yoshinaga ${ }^{2}$, Chietsugu Katoh ${ }^{3}$, Masanao Naya ${ }^{4}$, Robert A. deKemp ${ }^{5}$, and Nagara Tamaki ${ }^{1}$

${ }^{I}$ Department of Nuclear Medicine, Hokkaido University School of Medicine, Sapporo, Japan; ${ }^{2}$ Department of Molecular Imaging, Hokkaido University School of Medicine, Sapporo, Japan; ${ }^{3}$ Department of Health Sciences, Hokkaido University School of Medicine, Sapporo, Japan; ${ }^{4}$ Department of Cardiology, Hokkaido University School of Medicine, Sapporo, Japan; and ${ }^{5}$ Cardiac PET Centre, University of Ottawa Heart Institute, Ottawa, Ontario, Canada

The repeatability of rest and hyperemic myocardial blood flow (MBF) measurements using ${ }^{82} \mathrm{Rb}$ PET has not been evaluated. The aim of this study was to investigate the short-term repeatability of such measurements. Methods: Fifteen healthy volunteers underwent rest and pharmacologic stress ${ }^{82} \mathrm{Rb} \mathrm{PET}$, repeated 60 min apart. Results: There was no significant difference in repeated rest MBF $(0.77 \pm 0.25$ vs. $0.82 \pm 0.25 \mathrm{~mL} / \mathrm{min} / \mathrm{g}, P=$ 0.31 ; mean difference, $6.18 \% \pm 12.22 \%$ ) or repeated hyperemic $\operatorname{MBF}(3.35 \pm 1.37$ vs. $3.39 \pm 1.37 \mathrm{~mL} / \mathrm{min} / \mathrm{g}, P=0.81$; mean difference, $1.17 \% \pm 13.64 \%)$. The repeatability coefficients were $0.19 \mathrm{~mL} / \mathrm{min} / \mathrm{g}$ for rest MBF and $0.92 \mathrm{~mL} / \mathrm{min} / \mathrm{g}$ for hyperemia. Conclusion: MBF using ${ }^{82} \mathrm{Rb}$ is highly reproducible using a sameday short-term repeatability protocol. Serial MBF measurements with ${ }^{82} \mathrm{Rb}$ PET should have the ability to quantify the acute effects of therapeutic interventions on MBF.

Key Words: myocardial blood flow; pharmacologic stress; ${ }^{82} \mathrm{Rb}$

J Nucl Med 2009; 50:68-71

DOI: $10.2967 /$ jnumed.108.055673

A role for serial evaluation of patients to determine the response to therapy and progression of disease $(1,2)$.

${ }^{82} \mathrm{Rb} \mathrm{PET}$ has shown good diagnostic accuracy (3) and has prognostic value in patients with coronary artery disease (4). However, only limited data have been reported on myocardial blood flow (MBF) quantification, and there are no data evaluating repeatability using ${ }^{82} \mathrm{Rb}(5,6)$.

Establishing the reproducibility of ${ }^{82} \mathrm{Rb}$ MBF measurements is important for serial PET measurements of flow changes after various therapeutic interventions. The purpose of the current study was to investigate the same-day

Received Jul. 4, 2008; revision accepted Oct. 8, 2009.

For correspondence or reprints contact: Keiichiro Yoshinaga, Department of Molecular Imaging, Hokkaido University School of

Medicine, Kita15 Nishi7, Kita-Ku, Sapporo, Hokkaido, Japan 060-8638. E-mail: kyoshi@med.hokudai.ac.jp

COPYRIGHT @ 2009 by the Society of Nuclear Medicine, Inc. short-term repeatability of rest and hyperemic MBF as assessed by ${ }^{82} \mathrm{Rb}$ PET.

\section{MATERIALS AND METHODS}

\section{Study Protocol}

Fifteen healthy volunteers ( 8 men and 7 women) with a mean age $( \pm \mathrm{SD})$ of $29.4 \pm 9.3$ y participated.

MBF was measured at rest and with adenosine triphosphate stress $(7,8)$ using $1,480 \mathrm{MBq}$ of ${ }^{82} \mathrm{Rb}(4,5)$. Ten-minute dynamic scans were obtained using a Siemens HR+ PET scanner (5). Adenosine triphosphate $(160 \mu \mathrm{g} / \mathrm{kg} / \mathrm{min})$ was infused for $9 \mathrm{~min}$, and image acquisition was started 3 min after the beginning of the infusion (7).

MBF was measured first at rest and 10 min later during stress; then, both scans were repeated with a test-retest interval of $60 \min (9)$.

\section{Quantification of MBF}

MBF was measured using a previously described 1-tissuecompartment model (5).

The early-phase ${ }^{82} \mathrm{Rb}$ images were used to define a region of interest in the left ventricular blood pool. The myocardial uptake images were calculated by adding the late-phase data from 4 to 6 min. A whole-myocardium region of interest was positioned with an algorithm we have developed (8).

The following equation was used to estimate the inflow rate $\left(K_{1}\right)$ of ${ }^{82} \mathrm{Rb}$ into myocardium $\mathrm{Ct}(\mathrm{t})(5)$ :

$$
\mathrm{dCt}(\mathrm{t}) / \mathrm{dt}=K_{1} \cdot \mathrm{Ca}(\mathrm{t})-k_{2} \cdot \mathrm{Ct}(\mathrm{t})
$$

where $k_{2}$ is the outflow rate from myocardium into the blood $\mathrm{Ca}(\mathrm{t})$.

Conversion from $K_{1}$ to MBF was estimated with the modified Renkin-Crone model $(5,10)$.

Coronary flow reserve (CFR) was calculated as the ratio of MBF during stress to MBF at rest (2). The rest MBF was corrected for the rate-pressure product $(\mathrm{RPP})$ as rest $\mathrm{MBF} \times($ normal mean RPP/individual RPP) (7).

The normal mean RPP at rest in our institution was 8,150 . RPP-corrected CFR was calculated as stress MBF/RPP-corrected rest $\mathrm{MBF}$. 


\section{Statistical Analysis}

The percentage difference between the first and second studies was calculated as (second MBF - first MBF)/mean MBF $\times 100 \%$. A $P$ value of less than 0.05 was considered statistically significant.

\section{RESULTS}

\section{Physiologic Responses}

In total, 13 of $15(86.7 \%)$ participants had some adverse effects of adenosine triphosphate, but none of the participants had ischemic electrocardiography changes or chest pain.

The hemodynamic data did not significantly differ between the first and second studies (Fig. 1).

\section{MBF and CFR}

The repeatability coefficient of rest $\mathrm{MBF}$ was 0.19 $\mathrm{mL} / \mathrm{min} / \mathrm{g}$, hyperemic MBF was $0.92 \mathrm{~mL} / \mathrm{min} / \mathrm{g}$, and CFR was $1.61 \mathrm{~mL} / \mathrm{min} / \mathrm{g}$ (Table 1; Figs. 2 and 3). A good correlation was found between the first and second rest $\operatorname{MBF}(r=0.93, P<0.0001)$, hyperemic MBF $(r=0.94$, $P<0.0001)$, and CFR $(r=0.86, P<0.0001)$ (Fig. 4). RPP correction did not improve the repeatability of rest $\mathrm{MBF}(P=$ $0.08)$ or CFR measurements $(P=0.21)$.

Both men and women had good repeatability of rest MBF and hyperemic MBF (Table 1).

\section{DISCUSSION}

To the best of our knowledge, this was the first study to evaluate short-term MBF repeatability using generatorproduced ${ }^{82} \mathrm{Rb}$ PET. Our study demonstrated that ${ }^{82} \mathrm{Rb}$ PET rest and hyperemic MBF measurements and CFR had good repeatability. PET can quantify MBF and CFR using a suitable tracer kinetic model. In the current study, we applied a 1-tissue-compartment model for ${ }^{82} \mathrm{Rb} \mathrm{MBF}$

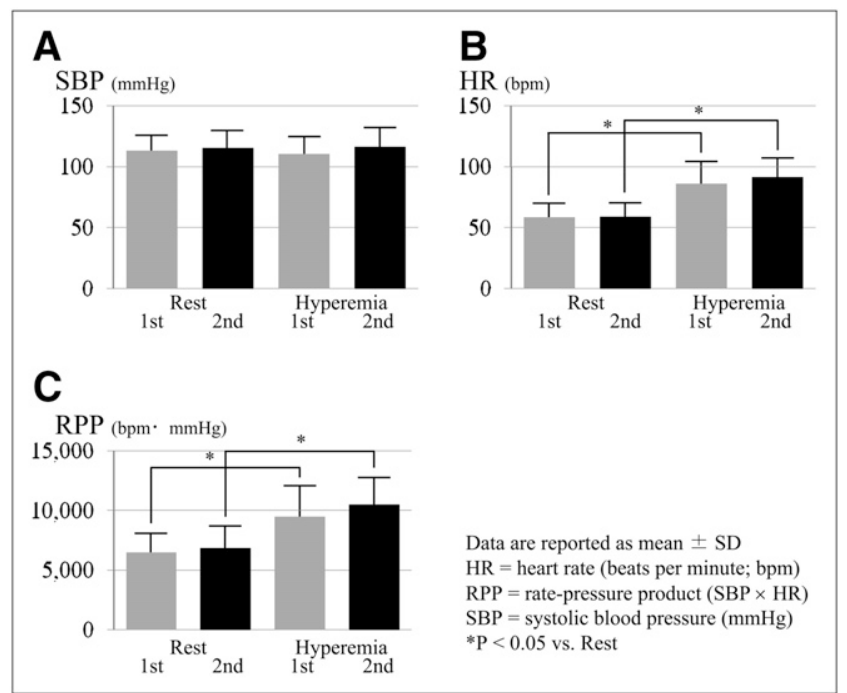

FIGURE 1. Hemodynamic data at first and second studies: systolic blood pressure at rest and during hyperemia (A), heart rate at rest and during hyperemia $(B)$, and rate-pressure product at rest and during hyperemia $(\mathrm{C})$.

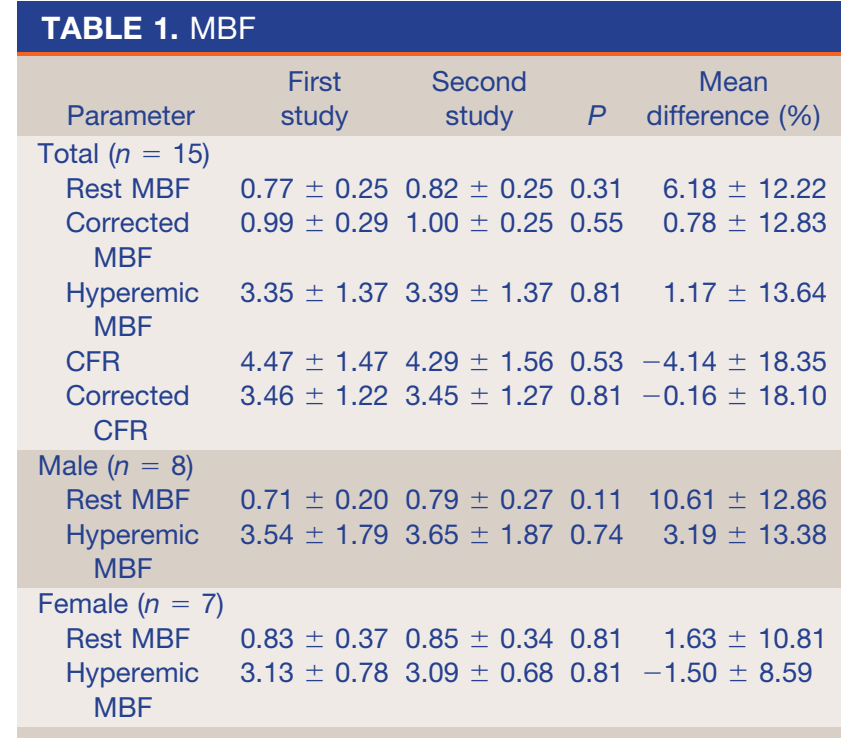

Data are reported as mean $\pm \mathrm{SD}$. Corrected MBF $=$ rest MBF $\times$ (normal mean RPP/individual RPP); MBF is expressed in $\mathrm{mL} / \mathrm{min} / \mathrm{g}$.

quantification similar to that of Lortie et al. (5), who reported good correlation with ${ }^{13} \mathrm{~N}$-ammonia. Our rest and hyperemic MBF values were similar to those of the previous study $(5,9,10)$, indicating that appropriate mathematic modeling was applied (7). It is known that there are sex differences in MBF (11), but in the present study both men and women had similar repeatability. Thus, this technique is applicable to both sexes.

${ }^{82} \mathrm{Rb}$ PET has several advantages. Myocardial perfusion images can be obtained without a cyclotron and with a short

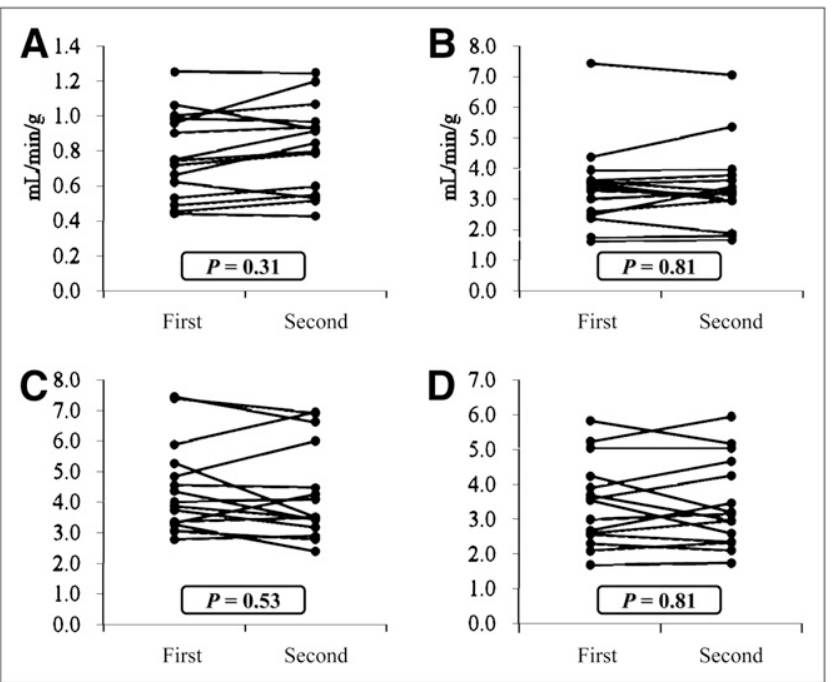

FIGURE 2. MBF and CFR comparing first and second studies: repeated rest $M B F(A)$, repeated hyperemic MBF (B), repeated CFR (C), and repeated RPP-corrected CFR (D). 
FIGURE 3. Repeatability of MBF Bland-Altman plots: rest MBF (A), RPP-corrected rest MBF $(B)$, hyperemic MBF (C), CFR (D), and RPPcorrected CFR (E).
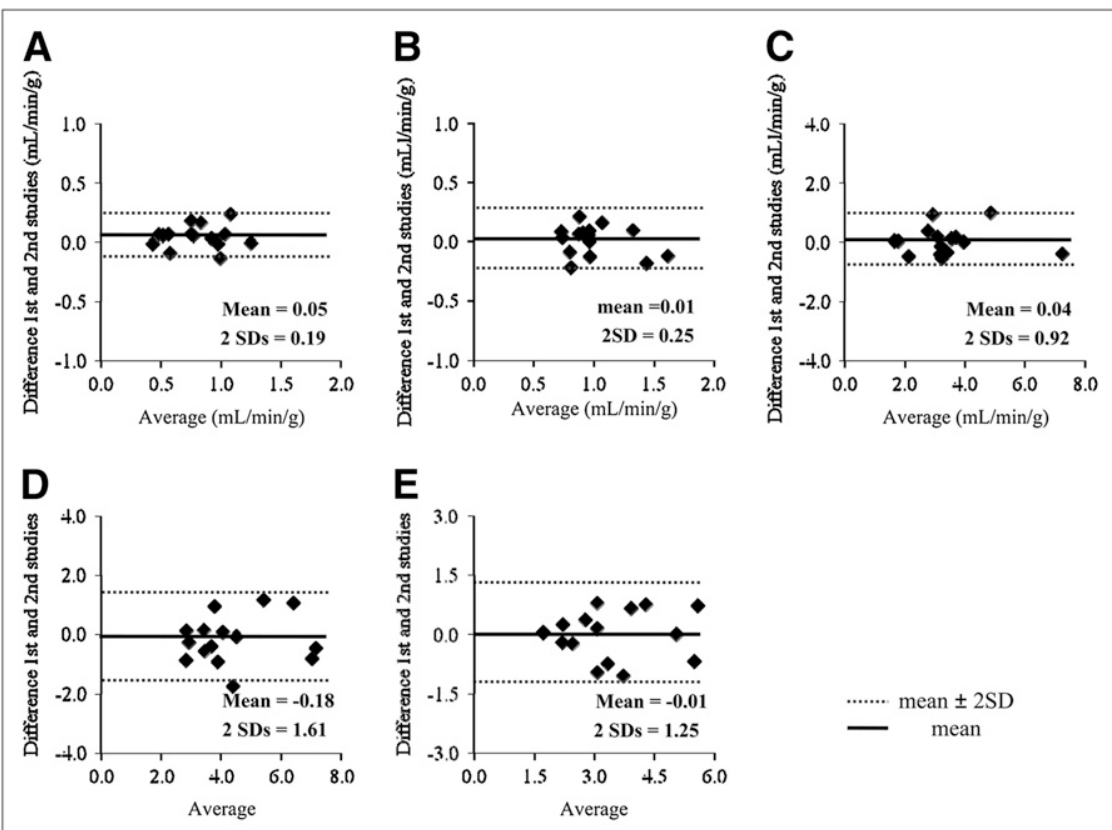

interval because of the short, 76-s, half-life (4). ${ }^{82} \mathrm{Rb} \mathrm{MBF}$ quantification may be useful for the evaluation of therapeutic interventions. However, as a first step, it is important to estimate the reproducibility of the technique as reported in the present study $(9,12)$.

The small mean difference in rest MBF in the current study indicated good repeatability and was slightly better than the previous findings of Kaufmann et al. using ${ }^{15} \mathrm{O}$ water (9) or of Nagamachi et al. using ${ }^{13} \mathrm{~N}$-ammonia (12). Rest MBF is considered to be associated with the RPP. Nagamachi et al. reported that RPP correction significantly reduced MBF variability. However, there was no significant change after RPP correction in the present study because the initial data had sufficiently low variability.

Hyperemic MBF in the current study showed small differences between the 2 studies indicating good repeatability as well. Compared with the previous studies by Kaufmann et al. (9) or Nagamachi et al. (12), the present study showed a smaller difference and similar SD. The decreasing net extraction of ${ }^{82} \mathrm{Rb}$ with increasing MBF (13) may be expected to increase variability during hyperemia. However, this relationship was not observed in the present study.

Our study had some limitations. It assessed the reproducibility of MBF and CFR in the whole left ventricular myo-
FIGURE 4. Scatter plots comparing first and second MBF studies: rest MBF (A), RPP-corrected rest MBF (B), hyperemic MBF (C), CFR (D), and RPPcorrected CFR (E).
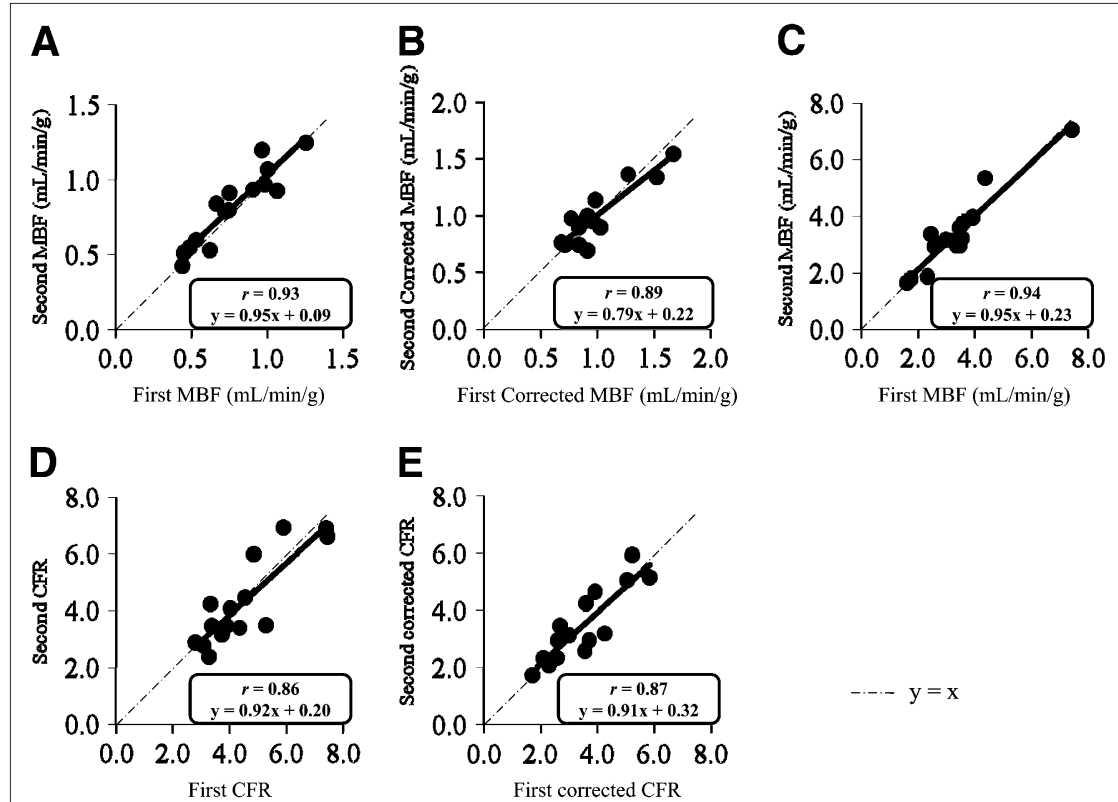

E

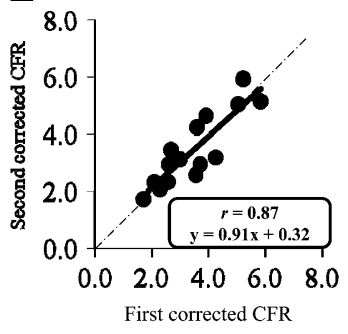


cardium but not in regional myocardial segments. Further studies are needed to evaluate reproducibility in patients with coronary artery disease, in whom additional regional heterogeneity may be expected. We studied only short-term repeatability, not repeatability over longer intervals. However, an advantage of ${ }^{82} \mathrm{Rb}$ is its short half-life, which enables various acute interventions to be evaluated in single-session studies similar to those using ${ }^{15} \mathrm{O}$-water. Thus, it is important to evaluate short-term repeatability in single-series studies (9). Nevertheless, additional prospective studies for longerterm reproducibility are warranted.

\section{CONCLUSION}

MBF and CFR using ${ }^{82} \mathrm{Rb}$ were highly reproducible in this study of same-day short-term repeatability.

\section{ACKNOWLEDGMENTS}

This study was supported in part by grant H19-C-068 from the Northern Advancement Center for Science and Technology (Sapporo, Japan).

\section{REFERENCES}

1. Machac J. Cardiac positron emission tomography imaging. Semin Nucl Med. 2005;35:17-36.

2. Yoshinaga K, Chow BJ, Dekemp RA, et al. Application of cardiac molecular imaging using positron emission tomography in evaluation of drug and therapeutics for cardiovascular disorders. Curr Pharm Des. 2005;11:903-932.
3. Bateman TM, Heller GV, McGhie AI, et al. Diagnostic accuracy of rest/stress ECG-gated Rb-82 myocardial perfusion PET: comparison with ECG-gated Tc99m sestamibi SPECT. J Nucl Cardiol. 2006;13:24-33.

4. Yoshinaga $\mathrm{K}$, Chow BJ, Williams $\mathrm{K}$, et al. What is the prognostic value of myocardial perfusion imaging using rubidium-82 positron emission tomography? J Am Coll Cardiol. 2006;48:1029-1039.

5. Lortie M, Beanlands RS, Yoshinaga K, Klein R, Dasilva JN, DeKemp RA. Quantification of myocardial blood flow with ${ }^{82} \mathrm{Rb}$ dynamic PET imaging. Eur J Nucl Med Mol Imaging. 2007;34:1765-1774.

6. Herrero P, Markham J, Shelton ME, Bergmann SR. Implementation and evaluation of a two-compartment model for quantification of myocardial perfusion with rubidium-82 and positron emission tomography. Circ Res. 1992;70: 496-507.

7. Yoshinaga K, Katoh C, Noriyasu K, et al. Reduction of coronary flow reserve in areas with and without ischemia on stress perfusion imaging in patients with coronary artery disease: a study using oxygen 15-labeled water PET. J Nucl Cardiol. 2003;10:275-283.

8. Katoh C, Morita K, Shiga T, Kubo N, Nakada K, Tamaki N. Improvement of algorithm for quantification of regional myocardial blood flow using ${ }^{15} \mathrm{O}$-water with PET. J Nucl Med. 2004;45:1908-1916.

9. Kaufmann PA, Gnecchi-Ruscone T, Yap JT, Rimoldi O, Camici PG. Assessment of the reproducibility of baseline and hyperemic myocardial blood flow measurements with ${ }^{15} \mathrm{O}$-labeled water and PET. J Nucl Med. 1999;40:18481856.

10. Katoh C, Yoshinaga K, Manabe O, Tamaki N. New quantification algorithm of regional myocardial blood flow with ${ }^{82}$ rubidium PET [abstract]. J Nucl Med. 2008;49(suppl 1):384P.

11. Chareonthaitawee P, Kaufmann PA, Rimoldi O, Camici PG. Heterogeneity of resting and hyperemic myocardial blood flow in healthy humans. Cardiovasc Res. 2001;50:151-161.

12. Nagamachi S, Czernin J, Kim AS, et al. Reproducibility of measurements of regional resting and hyperemic myocardial blood flow assessed with PET. J Nucl Med. 1996;37:1626-1631.

13. Huang SC, Williams BA, Krivokapich J, Araujo L, Phelps ME, Schelbert HR. Rabbit myocardial ${ }^{82} \mathrm{Rb}$ kinetics and a compartmental model for blood flow estimation. Am J Physiol. 1989;256:H1156-H1164. 